\title{
ON LEVI'S THEOREM FOR LEIBNIZ ALGEBRAS
}

\author{
DONALD W. BARNES
}

(Received 22 September 2011)

\begin{abstract}
A Lie algebra over a field of characteristic 0 splits over its soluble radical and all complements are conjugate. I show that the splitting theorem extends to Leibniz algebras but that the conjugacy theorem does not.
\end{abstract}

2010 Mathematics subject classification: primary 17A32.

Keywords and phrases: Leibniz algebras, Levi's theorem.

A left Leibniz algebra is a linear algebra $L$ whose left multiplication operators $d_{a}: L \rightarrow L$, defined by $d_{a}(x)=a x$ for all $a, x \in L$, are derivations. The basic theory of Leibniz algebras and their modules is set out in Loday and Pirashvili [3].

The subspace $\left\langle x^{2} \mid x \in L\right\rangle$ spanned by the squares of elements of $L$ is called the Leibniz kernel of $L$ and denoted $\operatorname{Leib}(L)$. It is an abelian ideal of $L, \operatorname{Leib}(L) L=0$ and $L / \operatorname{Leib}(L)$ is a Lie algebra. Thus, Leibniz algebras are almost Lie algebras and it is natural to consider which of the theorems about Lie algebras generalise to Leibniz algebras. For example, Ayupov and Omirov [1, Theorem 2] and Patsourakos [4, Theorem 7] have shown that Engel's theorem holds for Leibniz algebras. Ayupov and Omirov [1, Theorem 3] have shown that, if $L$ is a finite-dimensional Leibniz algebra over a field of characteristic 0 and $R$ is its soluble radical, then $L R$ is nilpotent.

The Levi-Malcev theorem asserts that, if $L$ is a finite-dimensional Lie algebra over a field of characteristic 0 , then $L$ splits over its soluble radical and that all complements are conjugate. In this note, I show that the splitting theorem extends to Leibniz algebras but that the conjugacy theorem does not. As for Lie algebras, the splitting theorem achieves some reduction of the investigation of representations of Leibniz algebras to problems of Leibniz representations of semi-simple Lie algebras and of representations of soluble Leibniz algebras.

THEOREM 1. Let L be a finite-dimensional Leibniz algebra over a field of characteristic 0 and let $R$ be its soluble radical. There exists a semi-simple subalgebra $S$ of $L$ such that $S+R=L$ and $S \cap R=0$.

(C) 2011 Australian Mathematical Publishing Association Inc. 0004-9727/2011 \$16.00 
Proof. Put $K=\operatorname{Leib}(L)$. Clearly, $K \subseteq R$ and $L / R$ is a semi-simple Lie algebra. By Levi's theorem (see Jacobson [2, Ch. III, p. 91]), there exists a semi-simple subalgebra $S^{*} / K$ of $L / K$ such that $S^{*}+R=L$ and $S^{*} \cap R=K$. It is sufficient to prove that $S^{*}$ splits over $K$, so we may suppose $R=K$.

Since $K L=0, L$ may be considered as a left module for the semi-simple Lie algebra $L / K$. By Whitehead's theorem (see Jacobson [2, Ch. III, Theorem 8, p. 79]), this module is completely reducible. Thus, there exists a submodule $S$ complementing $K$. Since $L S \subseteq S$, we have $S S \subseteq S$ and $S$ is a subalgebra.

ExAmple 2. Let $S$ be a simple Lie algebra and let $K$ be isomorphic to $S$ as a left $S$-module. I denote by $x^{\prime}$ the element of $K$ corresponding to $x \in S$ under this isomorphism. I make $K$ into a Leibniz module by defining the right action to be 0 . Let $L$ be the split extension of $K$ by $S$. Then $L$ is a Leibniz algebra and $\operatorname{Leib}(L)=K$. The space $S_{1}=\left\{\left(s, s^{\prime}\right) \mid s \in S\right\}$ is another subalgebra complementing $K$ since, using the module isomorphism,

$$
\left(s, s^{\prime}\right)\left(t, t^{\prime}\right)=\left(s t, s t^{\prime}\right)=\left(s t,(s t)^{\prime}\right) .
$$

For any $x=(s, k) \in L$, the inner derivation $d_{x}=d_{s}, d_{x}(S) \subseteq S$ and so $\exp \left(d_{x}\right)(S) \subseteq S$. Thus, $S$ and $S_{1}$ are not conjugate.

\section{References}

[1] Sh. A. Ayupov and B. A. Omirov, 'On Leibniz algebras', in: Algebra and Operators Theory, Proceedings of the Colloquium in Tashkent (Kluwer, Dordrecht, 1998), pp. 1-13.

[2] N. Jacobson, Lie Algebras (Interscience, New York-London, 1962).

[3] J.-L. Loday and T. Pirashvili, 'Leibniz representations of Lie algebras', J. Algebra 181 (1996), 414-425.

[4] A. Patsourakos, 'On nilpotent properties of Leibniz algebras', Comm. Algebra 35 (2007), $3828-3834$.

DONALD W. BARNES, 1 Little Wonga Rd, Cremorne, NSW 2090, Australia e-mail: donwb@iprimus.com.au 